\title{
Biochemical Studies on the Molecular Level of Polioviruses
}

\author{
Hend El Sherbini ${ }^{1}$, Naglaa Abd EI Meguid ${ }^{2}$ \\ ${ }^{1}$ Clinical Pathology Department, Faculty of Medicine, Cairo University \\ ${ }^{2}$ The Holding Company for Biological Products and Vaccines (VACSERA)
}

\begin{abstract}
Paralytic poliomyelitis continues to be an important disease affecting many children. The strategy for poliomyelitis eradication includes routine immunization, surveillance of acute flaccid paralysis (AFP) and mopping-up activities. Environmental monitoring, mainly involving the screening of waste water can provide evidence of the circulation or absence of wild-type polioviruses. The present work has been carried out to investigate the sequence divergence of neurovirulent derivatives of the Sabin oral poliovirus vaccine (OPV) strains, to establish epidemiological link between isolates, and to determine poliovirus genotype which circulates in Egypt. Enzyme Linked Immunoassay (ELIZA) and Reverse Transcription Polymerase Chain Reaction (RT-PCR) for intratypic differentiation have been used. Molecular analysis of the VPI/2A junction region has been performed on 22 isolates using the dideoxy chain termination method. Sequencing of the amplified region has been performed using the automated genetic analyzer ABI prism 310. Forward and reverse electropherograms were compared using sequencing alignment software version 2. Phylogenetic analysis of the VPI/2A region was done using Clustal $X$ software.

Key words: Sequence divergence, neurovirulent, epidemiological link, genotype, $\mathrm{VPl} / 2 \mathrm{~A}$ junction region, phylogenetic analysis.
\end{abstract}

\section{INTRODUCTION}

Poliomyelitis is a disease of great antiquity. Perhaps the earliest description is evident in an Egyptian stele from around $1350 \mathrm{BC}$ depicting a young man with typical asymmetric flaccid paralysis and atrophy of the leg. Several reports of the disease also appeared in the literature from the $17^{\text {th }}$ and $18^{\text {th }}$ centuries. By the mid $19^{\text {th }}$ century, the industrial revolution had brought increased urbanization to Europe and North America, changes and improvements in living conditions. Coincident with these massive changes was the advent of larger and more frequent outbreaks of poliomyelitis. From the late 1800 s, outbreaks were occurring in several European countries and in the United States $^{(\mathbf{1})}$.

Poliomyelitis was established as a viral disease in 1909 when Landsteiner and proper transmitted paralytic disease to monkeys by inoculating them with filtered stool from a patient with paralytic disease $^{(2)}$.

The major breakthrough for diagnosis and controlling poliomyelitis was the observation that poliovirus could be propagated in human embryonic tissues in culture ${ }^{(3)}$. 
These tissue cultures allowed easy isolation of the viruses and were prerequisite for the development of vaccines, including both the inactivated vaccine of Salk and the attenuated (oral) vaccine of Sabin ${ }^{(2)}$.

The genus Enterovirus is one of four genera belonging to the family Picornaviridae (small ribonucleic acid (RNA) viruses). The enteroviruses, grouped together and named in 1957, acquired their names since they infect the gastrointestinal tract. The genus enterovirus includes poliovirus (three serotypes), coxsackie A virus (23 serotypes including echovirus type 22 ), coxsackie B virus (six serotypes), echovirus (31 serotypes), and the human enteroviruses 68-71(4).

Enteroviruses are small RNA viruses consisting of a spherical, nonenveloped protein shell which encapsidate one molecule of singlestranded positive-sense RNA. The poliovirus particle has a diameter of 27 to $30 \mathrm{~nm}$. The virus capsid has an icosahedral symmetry and consists of 60 protomers. Each protomer comprises the four non-glycosylated virus proteins VP1 (34 kDa), VP2 (30kDa), VP3 $(27 \mathrm{kDa})$, and VP4 $(7.5 \mathrm{kDa})$. The virus proteins VP1, VP2 and VP3 are located at the capsid surface, whereas VP4 is located in the interior of the capsid in close contact with the viral RNA.

It has three antigenic types (serotypes 1, 2, and 3) whose physical properties are nearly identical. The different serotypes share between 36 and $52 \%$ of their nucleotide sequences. Neutralizing antibodies are directed towards VP1, VP2, and VP3, present in copies of 60 each of VP1 and VP3 and 58 to 59 copies of VP2 and VP4, containing at least four epitopes located on the surface of the virion. VP1 is the immuno-dominant antigen $^{(5)}$.

\section{Aim of the work}

Paralytic poliomyelitis continues to be important disease affecting many children. The strategy for poliomyelitis eradication includes routine immunization, surveillance of acute flaccid paralysis (AFP) and mopping-up activities. Environmental monitoring, mainly involving the screening of waste water can provide evidence of the circulation or absence of wild-type polioviruses. The ultimate goal of the polio eradication initiative is to stop polio vaccination. This can be only achieved by ensuring cessation of circulation of vaccine derived poliovirus (VDPVs), which were found to cause outbreaks of paralytic polio.

1. AFP surveillance for identification of divergent, neurovirulent derivatives of the Sabin oral poliovirus vaccine (OPV) strains.

2. Establishment of epidemiological links of the virus isolates from human samples, by comparing the nucleotide sequence of the outbreak isolates with each other and with the sequences of the other contemporary isolated polioviruses.

\section{MATERIALS \& METHODS}

\section{Study population:}

Virus strains used in the current study was isolated from 38 stool specimens collected from children suffering from AFP cases from 
different governorates in Egypt during January 2002 and February 2004. Patient's full data were recorded. They included patient's name, immunization status, number of doses, date of last OPV and onset date of AFP.

One stool sample was collected from a contact of a case number who died 2 days after the onset of paralysis. Three stool samples were collected from contact of case at one month interval between each sample. Seven stool samples were collected from a 14 years old patient suffering from AFP at interval 5, 36, 82, 140, 172,218 and 249 days after onset.

\section{Specimens processing:}

All fecal samples were treated with chloroform, to which enteroviruses are resistant as recommended by $\mathrm{WHO}^{(\mathbf{6})}$

B. Preparation of fecal samples for virus isolation

\section{Isolation of polioviruses:}

a. L20B cells, a mouse cell line (Lcells), genetically engineered to express the human poliovirus receptor.

b. $R D$ cells, derived from a human rhabdomyosarcoma

4. Identification of poliovirus isolates

Principle:

For the identification of poliovirus isolates, samples of diluted isolate with phosphate buffered saline (PBS), are mixed with equal volumes of a selected set of polyclonal antisera made in animals against poliovirus types 1, 2 and 3. Dilutions of $10^{-3}$ and $10^{-4}$ have been selected for use in that test expecting to contain $100 \mathrm{CCID}_{50}$ /50 $\mu \mathrm{l}$ of virus isolate and are mixed with high-titred polyclonal antisera. A back titration of the isolate is included in each test to allow calculation of the titre of virus actually present in that test $^{(\mathbf{6})}$

\section{Intratypic differentiation of} polioviruses:

Isolation and intratypic identification (ITD) of polioviruses from AFP cases is the first step in detecting the circulation of wild polioviruses and VDPV in the community. Vaccine strains circulate widely during and after immunization campaigns and may be found in healthy and symptomatic children. It is therefore necessary to determine whether poliovirus isolates are wild or vaccine-derived. Two methods were used for ITD, Enzyme Linked Immunosorbant Assay (ELISA) and diagnostic Reverse transcription Polymerase Chain Reaction (RTPCR).

a. ELISA method for intratypic differentiation $^{(7)}$

b. 2- RT-PCR for intratypic differentiation $^{(7)}$

Reverse transcription-PCR was performed on identified typed polioviruses. The viral RNA was released by heat and converted to complementary deoxyribonucleic acid (cDNA) using reverse transcriptase. The cDNA was amplified in a conventional PCR reaction using Taq polymerase. The PCR products were resolved by gel electrophoresis to determine size and function as confirmation of product authenticity. Both the cDNA synthesis and the PCR reaction use multiple sets of oligonucleotide primers with different specificities. Primers include Sabin type specific for each of the three serotypes and primers that amplify all 
polioviruses and all isolates of each of the three serotypes. Such combination of primers will result in characterization of polio isolates and will also confirm the serotype identification of the isolate.

\section{RESULTS}

Demographic characteristics of the study population:

The age ranged from 4 months to 14 years with a mean of $2.28 \pm 2.29$ years. They were 24 boys $(58.53 \%)$ and 17 girls $(41.46 \%)$, the male to female ratio was 1.5: 1.06 .

Geographical distribution:

Poliovirus was detected in 21 cases (51.21\%) from Fayoum, Benisuef, Menya, Souhag, Assuit and Kena (Upper Egypt), in 14 cases (34.14\%) from Behira, Kalyoubia, Menoufia, Dakahlia, Alexandria, Sharkia and Kafr El-Sheikh (Lower Egypt) and in 6 cases (14.63\%) from Cairo and Giza (Greater Cairo) (figure 1).

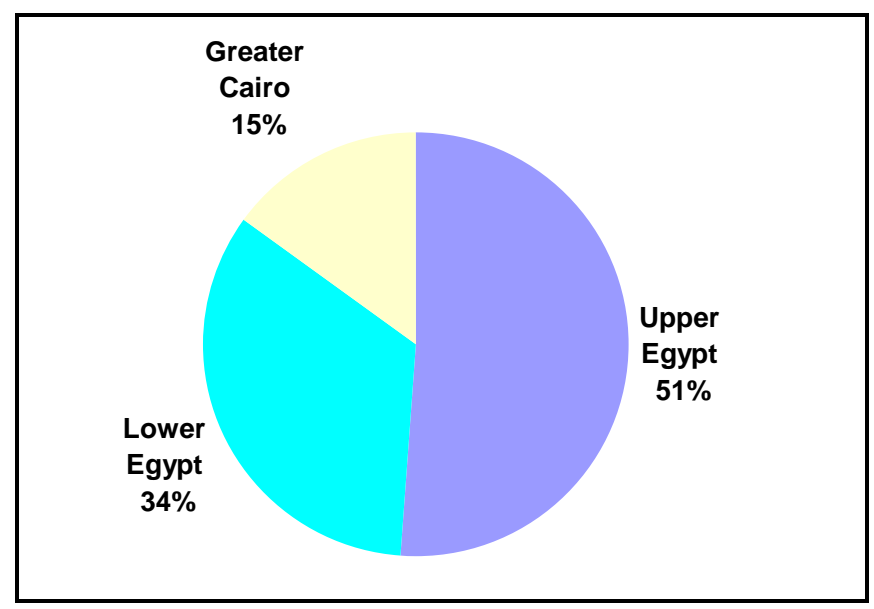

Figure (1): Geographical distribution of poliovirus in Egypt

Prevalence of isolation of each serotype post vaccination:

PV3 was isolated from 11 cases within group 1 (37.93\%), PV1 was isolated from 12 cases (41.38\%), PV2 was isolated from 3 cases $(10.34 \%)$ and mixture of PV2 and PV3 was isolated from 2 cases $(6.9 \%)$. One case $(3.45 \%)$ developed AFP post vaccination with no virus shedding. Virus identification:

Virus isolation and identification using specific antisera revealed that
$18(43.90 \%)$ of cases were poliovirus type $1 ; 5$ cases $(12.2 \%)$ were poliovirus type 2 and 14 cases $(34.15 \%)$ were poliovirus type 3 . mixture of poliovirus type 2 and poliovirus type 3 was found in 3 cases (7.32\%).

Intratypic differentiation was done by two different methods:

1. Antigenic method (ELISA) and isolates were characterized as:

Eighteen cases were poliovirus type 1: 
- 9 out $18(50 \%)$ were non Sabin poliovirus type 1 (NSL1).

- 9 out $18(50 \%)$ was Sabin like poliovirus type 1 (SL1).

- 5 cases were Sabin like (SL) poliovirus type 2, 14 cases were SL poliovirus type 3 and 3 cases were SL2 and SL3.

2. RT-PCR:

- Poliovirus strains were subjected to RT-PCR with pan poliovirus primer specific bands of $79 \mathrm{bp}$ were detected (fig. 2)
- 18 isolates were positive using PV serotype 1 primer

- 5 isolates were positive using PV serotype 2 primer

- 14 isolates were positive using PV serotype 3 primer

- With multiplex primer, 8 isolates were SL1, 5 isolates were SL2, 14 isolates were SL3 and 3 isolates were a mixture of SL2 and SL3.

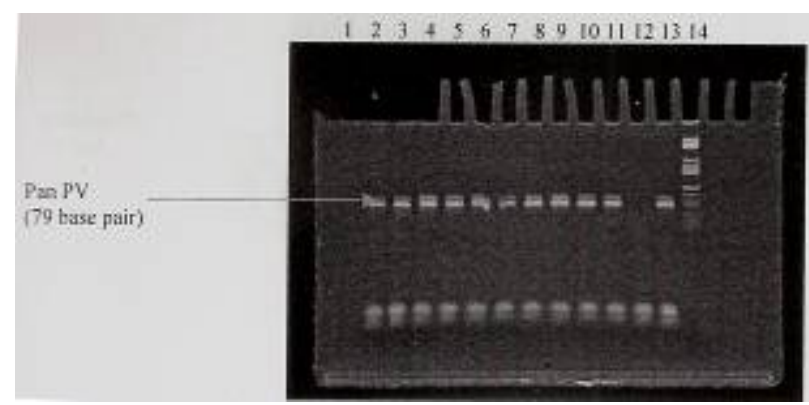

Figure (2): Pan poliovirus specific amplification of isolates Lane 2 to 11 samples, Lane \# 12: negative control Lane \# 13: positive control and lane \# 14 Marker

\section{Sequencing of amplified product}

Analysis of the sequencing of the VP1/2A region (295bp) has been performed on 22 available isolates and showed that 4 isolates were SL1, 3 isolates were SL2, 6 isolates were SL3 and 9 isolates were wild poliovirus type 1.

Genotypic relationship among poliovirus type I strains:

To determine the genotype of wild type 1 poliovirus, we compared our 9 sequences with wild type 1 sequences isolated from South Asia genotype $(\mathrm{SOAS})^{\mathbf{( 8 )}}$, from West Africa genotype (WEAF), from Northeastern Africa and Western Asia genotype
$(\mathrm{NEAF})^{(\mathbf{9 , 1 0})}$ using the clustal $\mathrm{X}$ program. We found that our isolates were genotype NEAF. Our isolates showed high degree of similarity of more than 93\% with strains isolated from Egypt from 1998 to 2001, and showed similarity ranging from 85 to $88 \%$ with strains isolated from Egypt in 1992 and from Israel in 1987 and 1988.

Within the isolates, a high percentage of genetic similarity was found. The strain isolated from Assuit in May 2004 differed by 3\% from that isolated from Menoufia in September 2002. The strain isolated from Alexandria in December 2002 differed 
by $4 \%$ from the strain isolated from Giza in October 2002. The strain isolated from Sharkia in December 2002 differed by $5 \%$ from that isolated from Belbies in November
2002. No nucleotide difference was obtained when sequential samples from case number 14 and case number 41 were analyzed (figure 3 ).

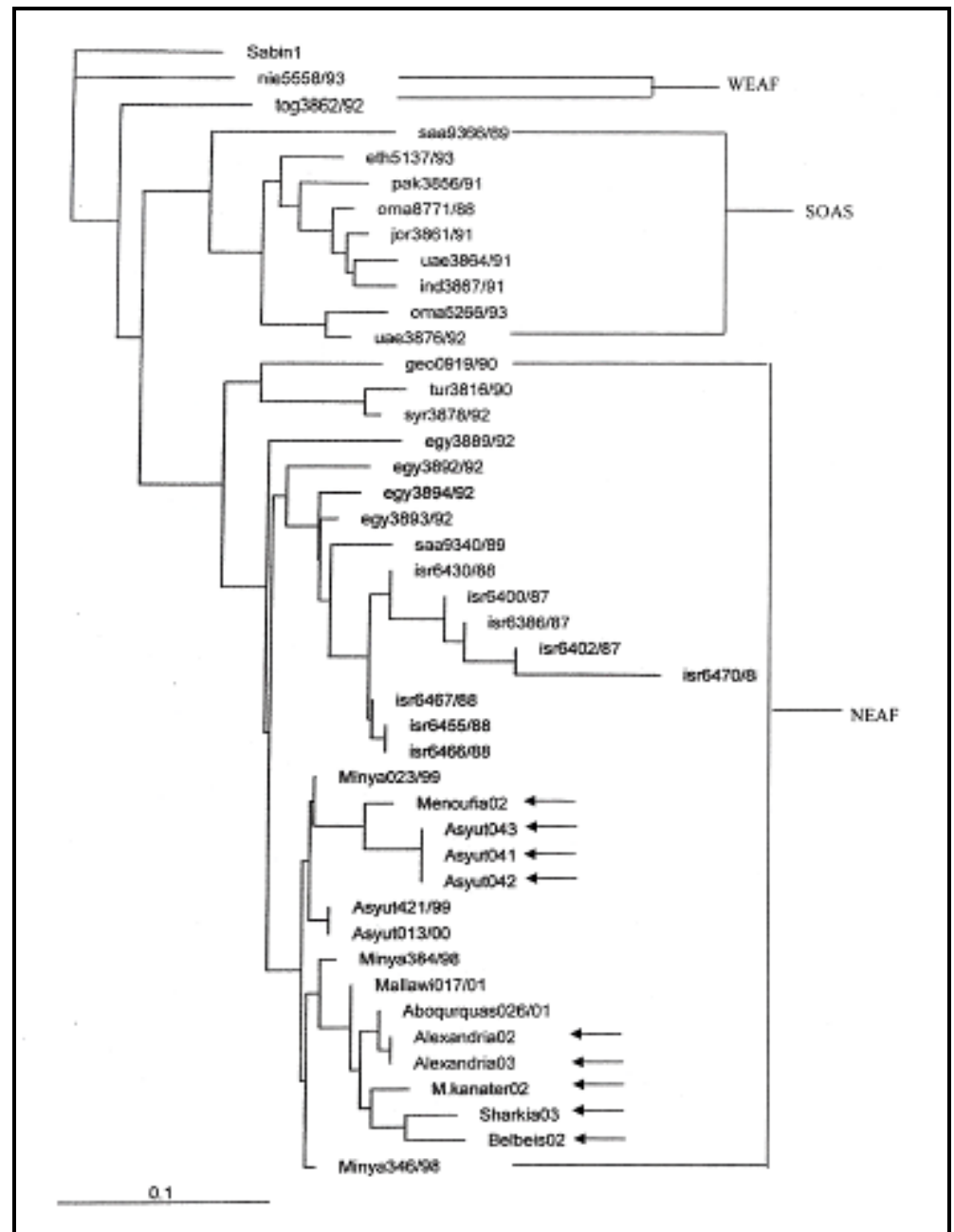

Figure (3): Phylogenetic tree summarizing sequence relationship among wild type 1 polioviruses isolated in Egypt from 2002 to 2004 (arrows) and strains from the Middle East, Africa and other region based on analysis of the 150 nucleotide within the VP/2A junction. The tree was rooted to the sequence of Sabin strain.

Country abbreviation. Nie: Nigeria; tog: Togo; saa: Saudi Arabia; eth: Ethiopia; Pak: Pakistan; Oma: Oman; Jor: Jordan; uae: United Arab Emirates; ind: India; geo: Georgia; Tur: Turkey; Syr: Syria; egy: Egypt; isr: Israel 


\section{DISCUSSION}

Paralytic poliomyelitis has been known for at least 3500 years and continues to be serious threat to children in parts of Asia, Africa and the Middle East. Poliovirus infects and replicates in the intestinal tract. Over $90 \%$ of infections are unapparent, but a viremia can develop and cause AFP usually 7-14 days after infection. The site of the paralysis depends on the location of the nerve cells destruction. The paralysis is typically asymmetric and reaches its maximum extent within 3-4 days. Any paralysis still present after 60 days is likely to be permanent. Humans are the only reservoir of poliovirus and infection is transmitted by the oralfecal route and less commonly by pharyngeal secretion ${ }^{(11)}$

In 1988, the WHO passed a resolution that aimed for global eradication of poliomyelitis. The key eradication strategies implemented by the WHO include high routine OPV coverage, synchronized immunization campaigns through the national immunization days (NIDs), intensified immunization in high risk areas and surveillance for AFP cases. In 2003, there were approximately 2000 reported cases worldwide and polio was endemic in only seven countries Afghanistan, Egypt, India, Niger, Nigeria, Pakistan and Somalia) ${ }^{(\mathbf{1 2})}$.

In 2005, an outbreak was reported in Yemen with confirmed 302 cases $^{(13)}$, laboratory investigation confirmed that the virus is very closely related to wild poliovirus currently circulating in Sudan. Second outbreak was detected in Indonesia with 122 reported cases; genetic analysis of the virus demonstrates that its origin is in West Africa, similar to the viruses which caused an outbreak in 2003-2004 in that region. Further analysis suggests the virus traveled to Indonesia through Sudan, and is similar to recently isolated viruses in Saudi Arabia and Yemen. Prior to that outbreak, Indonesia had been poliofree since $1995^{(\mathbf{1 4})}$

The third outbreak occurred in Angola, which had been polio-free since 2001, with 2 reported polio cases. Genetic sequencing shows that it originated in India, epidemiological evidence suggests a recent importation $^{(15)}$

Despite the many advantages of OPV, the most serious complication of its use is Vaccine associated paralytic poliomyelitis (VAPP) which is associated with reversion of the attenuated virus to its neurovirulent form ${ }^{(16)}$. Compared with immunocompetent children, the risk of VAPP is almost 7,000 times higher for persons with certain types of immunodeficiencies, particularly $\mathrm{B}$ lymphocyte disorders (e.g., agammaglobulinemia and hypogamma-globulinemia).

The risk of VAPP was not equal for all OPV doses in the vaccination series. The risk of VAPP is 7 to 21 times higher for the first dose than for any other dose in the OPV series. The risk of VAPP was one case per 1.4 million first doses. The risk for all other doses was one per 27.2 million doses. The reason for that difference by dose is not known with certainty, but is probably because the vaccine virus is able to replicate longer in a 
completely non-immune infant. That prolonged replication increases the chance of the emergence of a revertant virus that may cause paralysis. The situation is similar for contacts. A non-immune child may shed virus longer, increasing the chance of exposure of a contact ${ }^{(17)}$

According to $\mathrm{WHO}^{(\mathbf{1 8})}$, polioviruses was divided into three categories; "Sabin like viruses", the vast majority of vaccine related are "OPV-like", and have close sequence relationships (> 99\% VP1 sequence identity) to the original OPV strains. Immunologically normal OPV recipients usually excrete viruses for an average of three to four weeks. Short excretion periods and high population immunity normally limit the person to person spread of these OPV-like viruses. The second category "Vaccine-derived polioviruses" (VDPV) isolates show up to 99 to $85 \%$ VP1 sequence identity to the parental Sabin strains and the extent of their genetic changes is indicative of prolonged replication. The third category was "Non-Sabin like or wild polioviruses" with $>15 \%$ nucleotide sequence divergence from the Sabin serotype strain in VP1 region.

Archaeological evidence suggests that polio was present in Egypt at the dawn of civilization ${ }^{(\mathbf{1 9})}$. In the late 1970s, all three serotypes of indigenous wild poliovirus were circulating in Egypt ${ }^{(9)}$. The last case wild type 2 poliovirus from Egypt was isolated in 1979. Wild poliovirus type 3 was last detected in December 2000; wild type 1 was repeatedly isolated in Egypt in the southern governorates of Minya, Assuit, Sohag and Kena in
2001 and 2002 from both clinical specimens and environmental samples $^{(\mathbf{2 0 , 2 1 )}}$.

The partial sequence information of the 150 bases encoding parts of the capsid protein VP1 and the non-capsid protein $2 \mathrm{~A}$ allow to identify numerous geographic foci of endemic circulation of polioviruses, reveal previously unsuspected links between cases in distant communities, monitor the displacement from the environment of preexisting polioviruses by viruses from other regions and recognize the recombinant (vaccine-wild; wild-wild) origins of some epidemic polioviruses $^{(22)}$.

These molecular methods are based on the rapid evolution of the poliovirus genome during replication in the human gut. An estimated 1 to $2 \%$ of the total genome changes per year at a rate of 1-2 fixed nucleotide substitutions per week.

Most nucleotide substitutions are silent mutations, not causing changes in amino acid composition. The estimation of an epidemiologic link is based on this rapid evolution of poliovirus genomes by study of the patterns of nucleotide variation ${ }^{(\mathbf{1 0})}$

In our study, poliovirus was isolated from 39 cases suffering from AFP and two contact cases. Our goals of the present study were to identify sequence divergence or neurovirulent derivatives of the Sabin OPV strains, to establish epidemiological link between isolates, and to determine poliovirus genotype which circulate in Egypt. Comparing the geographical distribution of polioviruses, $51.21 \%$ of cases were from Upper Egypt, 34.14\% of cases were from lower Egypt and $14.63 \%$ were from Greater Cairo. All 
patients had received OPV and were classified as having VAPP according to WHO classification criteria $^{(\mathbf{2 3})}$. OPV derived strains were isolated from 31 cases $(75.61 \%)$ who presented a poliomyelitis syndrome with AFP, while wild poliovirus was detected in 9 cases (21.95) and no virus was isolated from one case $(2.44 \%)$

Our findings reveal that the incidence of AFP cases post vaccination due to type 1 was $41.38 \%$, type 2 was $10.34 \%$, type three was $37.93 \%$, mixture of type 2 and type 3 was $6.90 \%$ and one $(3.45 \%)$ developed AFP post vaccination with no virus shedding. Study done by Strebel et al. ${ }^{(24)}$, showed that the incidence of VAPP cases due to type 1 vaccine derived poliovirus is significantly lower than that for type 3 or type 2 virus, suggesting that the type 1 vaccine strain has the most stable attenuated phenotype.

In our study, patient number 14 stopped poliovirus shedding after 12 weeks, while patient number 41 excreted the virus for 9 weeks. No nucleotide difference was obtained when sequential samples of case number 14 and case number 41 were analyzed. Study done by Triki et al., on the duration of poliovirus excretion showed that virus was excreted during at least 7 weeks $^{(25)}$, while study by Alexander et al. ${ }^{(26)}$, showed that fecal excretion of vaccine viruses continues beyond the fifth week in less than $20 \%$ of immunized individuals

Intratypic differentiation using both the antigenic ELISA method and the molecular PCR method revealed that Sabin 3 was isolated from 34.15 $\%$ of cases, Sabin 1 was isolated from
$21.95 \%$ of cases, Sabin 2 was isolated from $12.2 \%$ of cases and mixture of type 2 and type 3 were isolated from $7.32 \%$. Wild poliovirus 1 was isolated from $21.95 \%$ of cases.

Our results showed that the incidence of Wild Poliovirus (WPV) type 1 decreased from 6 cases in 2002 (five from Lower Egypt and Greater Cairo and one from Upper Egypt) to one in 2003, reported in June from Abuqurqas district, Menya Governorate (Upper Egypt). It was isolated from a contact of an AFP patient in the same district in October 2003. In May 2004, one case was identified from Dairut district, Assuit Governorate (Upper Egypt, bordering Menya governorate). It was also isolated from one of the close contact of that patient. AFP patients and positive contacts had received OPV before paralysis onset.

Molecular analysis of the VP1/2A junction region has been performed on 22 isolates. Sequence analysis of vaccine derived strains revealed that they share more than $99 \%$ sequence identity with the original Sabin strain within the VP1/2A region and were classified as SL viruses.

We suggest that our patients were recently infected with the virus as demonstrated previously by Yang et al. ${ }^{(27)}$ and that the close sequence relationship is an indication of limited virus replication since administration of the initiating OPV dose. This finding also questions the immunological status of these AFP patients.

Given that a genotype is defined as a group of viruses showing at least $85 \%$ sequence similarity within the 150 nt $\mathrm{VPl} / 2 \mathrm{~A}$ region $^{(22)}$. The 
phylogenetic analysis of our wild type strains shows that they fall within a single genotype. The Wild Poliovirus (WPV) type 1 isolates were genotype NEAF as they were closely related to NEAF genotype isolated from Egypt from 1998 to 2001 with more than 93\% nucleotide similarity, with strains from Israel in 1987 and 1988 and with strains from Egypt in 1992 with similarity ranging from $85 \%$ to $88 \%$.

The phylogenetic tree also shows two groups of isolates one group of strains was found in Menoufia in 2002 and in Assuit in 2004, with 97\% nucleotide similarity. The other group of strains was found in Alexandria from 2002 to 2003 in Menshat-kanater and Belbeis, in 2002 and Sharkia in 2003 with nucleotide similarity more than $94 \%$. The two groups of strains differ by $12 \%$ nucleotide differences. The isolates from Egypt from 1998 to 2001 fall between these two groups. This finding suggests that at least two chains of transmission circulate in Egypt.

Only one confirmed WP1 case was reported in 2004. However, environmental surveillance indicates low level of persistent transmission of two separate lineages across the country, particularly in Cairo, Guiza (Greater Cairo), Assuit and Menya governorates (Upper Egypt). WP3 has not been found since December $2000^{(28)}$.

In conclusion, there is a remarked decrease in the incidence of Wild Poliovirus (WPV) type 1 from 6 cases in 2002 to one in 2003 and 2004. Only one genotype was circulating in Egypt with 2 chains of transmission. VDPV was not circulating in Egypt with improved OPV coverage. Further studies of poliovirus infection in immunodeficient patients and environmental specimens together with the genetic variation of the whole VP1 region should be considered.

\section{REFERENCES}

1. WHO (2004): Polio laboratory manual $4^{\text {th }}$ edition World Health Organization, Geneva.

2. Zeichhardt, H. and Grunert, $\mathbf{H}$. (2000): Enteroviruses. In "Clinical virology manual" Specter, S.; Hodinka, R.L. and Young, S.A. (eds.) $3^{\text {rd }}$ edition, American Society for microbiology press, Washington, D.c.p. 252-269.

3. Enders, J.F.; Weller, T.H. and Robbins, F.C. (1949): Cultivation of lansing strain of poliomyelitis virus in cultures of various human embryonic tisuue. Science, 109: 85-87.

4. Grandien, M.; Forsgren, M. and Ehrnst, A. (1999): Enteroviruses, In: Diagnostic procedures for viral, rickettsial, and chlamydial Infections, Lennette EH; Lennette DA and Lennette E, Health association pp 279-297.

5. Sutter R.; Cochi, S. and Melnick, J. (2003): Live attenuated poliovirus vaccines. In VACCINES Plotkin S.A. and Orenstein, W.A (eds.). $3^{\text {rd }}$ ed. W.B. Saunders company P. 651705.

6. WHO (2001b): Polio laboratory manual World Health Organization, Geneva.

7. Van der Avoort, HG.; Hull, BP.; Hovi, T.; Pallansch, MA.; 
Kew, OM.; Crainic, R.; Wood, DJ.; Mulders, MN. And Van Loon, AM. (1995): Comparative study of five methods for intratypic differentiation of polioviruses. J. Clin. Microbiol., 33 (10): 2562-2566.

8. Shulman, LM.; Handsher, R.; Yang, CF.; Yang, SJ.; Manor, J.; Vonsover, A.; Grossman, Z.; Pallansh, M.; Mendelson, E. and Kew, OM. (2000): Resolution of the pathways of poliovirus type 1 transmission during an outbreak J. Clin. Microbiol., 38 (3): 945-952.

9. Kew, OM.; Mulders, MN. And Lipskaya, GY. (1995): Molecular epidemiology of polioviruses. Semin. Virol., 6: 401-414.

10. Mulders, MN.; Lipskaya, GY.; Van der Avoort, HG.; Koopmans, MP.; Kew, OM. And van Loon, AM. (1995a): Molecular epidemiology of wild piliovirus type I in Europe, the Middle East, and the Indian subcontinent. J. Infect. Dis., 171 (6): 1399-1405.

11. Benenson, AS (1995): Control of communicable diseases manual. $16^{\text {th }}$ edition Washington: American public Health association.

12. Sangrujee, N.; Caceres, VM. and Cochi, SL. (2004): Cost analysis of post-polio certification immunization policies WHO Bulletin, 82 (1): 9-15.

13. Polio eradication situation report (2005): polio eradication Situation report-July 2005 www.polioeradication.org
14. WHO (2005c): Poliomyelitis outbreak spreads across Yemen; case confirmed in Indonesia. Weekly epidemiological record, 80 (18): 149-156.

15. WHO (2005d): Poliomyelitis, Angola Weekly epidemiological record, 80 (27): 233-240.

16. Guillot S.; Caro, V.; Cuervo, N.; Korotkova, E.; Combiescu, M.; Persu, A.; AubertCombiescu, A.; Delpeyroux, F. and Crainic, R. (2000): Natural genetic exchanges between vaccine and wild poliovirus strains in humans J. Virol., 74 (18): 8434-8443.

17. CDC (2000): Poliomyelitis. In Epidemiology \& Prevention of Vaccine-Preventable Diseases “The Pink Book" p. 89-95.

18. WHO (2001c): Network Strategy for detecting cVDPV is defined polio Lab network Quarterly Update, 7 (4): 1-4.

19. Paul, J. (1971): A history of poliomyelitis. Yale University Press, New Haven, conn.

20. WHO (2002): Progress towards poliomyelitis eradication. Egypt, 2001 Weekly epidemiological record, 77 (9): 69-76.

21. WHO (2003): Global Polio eradication initiative to launch strategic plan 2004-2008. Polio News, 20: 1-6.

22. Rico-Hesse, R.; Pallansch, MA.; Nottay, BK. and Kew, OM (1987): Geographic distribution of wild poliovirus type I genotypes virology, 160 (2): 311322.

23. WHO (1997): Combined immunization of infants with oral and inactivated poliovirus 
vaccines: results of a randomized trial in the Gambia, Oman, and Thailand. WHO Collaborative Study Group on Oral and inactivated Poliovirus Vaccines J. Infect. Dis., 175 (suppl 1): S215S227.

24. Strebel, PM.; Sutter, RW.; Cochi, SL.;Biellik, RJ.; Brink, EW.; Kew, OM.; Pallansch, MA.; Orenstein, WA. and Hinman, AR. (1992): Epidemiology of poliomyelitis in the United States one decade after the last reported case of indigenous wild virus-associated disease. Clin. Infect. Dis., 14(2): 568-579.

25. Triki, H.; Barbouche, MR.; Bahi, O., Bejaoui, M. and Dellagi, K. (2003): CommunityAcquired poliovirus infection in children with primary immunodeficiencies in Tunisia. J.
Clin. Microbiol., 41 (3): 12031211.

26. Alexander, JP Jr.; Gary, HE Jr. and Pallansch, MA (1997): Duration of poliovirus excretion and its implications for acute flaccid paralysis surveillance: a review of the literature. J. Infect. Dis., 175 (Suppl. 1): $\quad$ S176S182.

27. Yang, CF.; Naguib, T.; Yang, SJ.; Nasr, E.; Jorba, J.; Ahmed, N.; Campagnoli, R.; Van Der Avoort, H.; Shimizu, H.; Yoneyama, T.; Miyamura, T.; Pallanch, M. and Kew, $O$. (2003): Circulation of endemic type 2 vaccine-derived poliovirus in Egypt from 1983-1993. J. Virol., 77 (15): 8366-8377.

28. CDC (2004): Progress towards poliomyelitis eradication- Egypt, 2003-2004. Morb. Mortal. Wkly. Rep., 53 (35): 820-822.

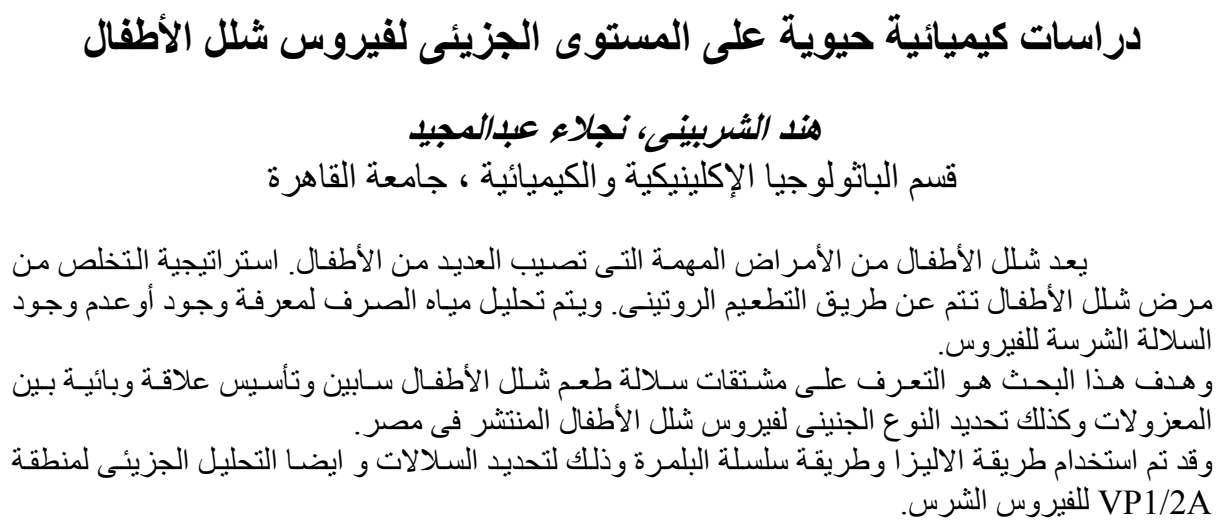

